\title{
Statutory Regulation of Traditional Medicine Practitioners and Practices: The Need for Distinct Policy Making Guidelines
}

\author{
Nadine ljaz, PhD, and Heather Boon, BScPhm, PhD
}

\begin{abstract}
The World Health Organization (WHO) has called for the increased statutory regulation of traditional and complementary medicine practitioners and practices, currently implemented in about half of nations surveyed. According to recent WHO data, however, the absence of policy guidelines in this area represents a significant barrier to implementation of such professional regulations. This commentary reviews several key challenges that distinguish the statutory regulation of traditional medicine practitioners and practices from biomedical professional regulation, providing a foundation for the development of policy making parameters in this area. Foremost in this regard are the ongoing impacts of the European colonial encounter, which reinforce biomedicine's disproportionate political dominance across the globe despite traditional medicine's ongoing widespread use (particularly in the global South). In this light, the authors discuss the conceptual and historical underpinnings of contemporary professional regulatory structures, the tensions between institutional and informal traditional medicine training pathways, and the policy challenges presented by the prospect of standardizing internally diverse indigenous healing approaches. Epistemic and evidentiary tensions, as well as the policy complexities surrounding the intersection of cultural and clinical considerations, present additional challenges to regulators. Conceptualizing professional regulation as an intellectual property claim under the law, the authors further consider what it means to protect traditional knowledge and prevent misappropriation in this context. Overall, the authors propose that innovative professional regulatory approaches are needed in this area to address safety, quality of care, and accessibility as key public interest concerns, while prioritizing the redress of historical inequities, protection of diverse indigenous knowledges, and delivery of care to underserved populations.
\end{abstract}

Keywords: traditional medicine, professional regulation, European colonization, equity, World Health Organization

$\mathbf{I}$ N ITs 2014 Traditional Medicine Strategy, the World Health Organization (WHO) recommends that nations take steps to regulate traditional and complementary medicine (T\&CM) "practices and practitioners." 1 Among the principles underlying the WHO's regulatory recommendations are the enhancement of "safety, quality, and effectiveness" in the delivery of T\&CM care and T\&CM's "global integration... into health systems." Such aims, the Strategy suggests, should be addressed with reference to "protect[ing] the intellectual property rights of indigenous people and local communities and their healthcare heritage."
This article-a commentary - aims to initiate a global conversation about the distinct challenges faced by policy makers in the area of T\&CM professional regulation. The authors do so by reviewing several key features that differentiate the statutory regulation of traditional medicine practitioners and practices (i.e., those rooted in particular indigenous knowledge systems) from biomedical professional regulation. Adopting a postcolonial theoretical lens, ${ }^{2}$ the authors point to factors arising historically from the European colonial encounter that continue to impact on regulatory matters across the globe today. To support their

Leslie Dan Faculty of Pharmacy, University of Toronto, Toronto, Canada.

(C) Nadine Ijaz and Heather Boon, 2018; Published by Mary Ann Liebert, Inc. This Open Access article is distributed under the terms of the Creative Commons Attribution Noncommercial License (http://creativecommons.org/licenses/by-nc/4.0), which permits any noncommercial use, distribution, and reproduction in any medium, provided the original authors and the source are cited. 
position, the authors use examples from their ongoing research in the Canadian context, augmented by global literature on T\&CM professional regulation. The article's goal is to identify conceptual parameters that will catalyze a global conversation as opposed to taking an exhaustive approach in the examples provided. Before turning to their core argument, the authors provide some clarification about terminology.

It should be noted that some traditional medicine practices-such as acupuncture-have been incorporated into biomedical practitioners' scopes in some jurisdictions, where these practices are termed complementary medicine. Other so-called complementary medicine practices, such as chiropractic and osteopathy, do not have precolonial, indigenous cultural roots (although many other indigenous medicine practices include types of massage and manual techniques), but are distinguished from conventional care by their relative marginality within biomedically dominant healthcare systems. Although the arguments presented in what follows may have some relevance for the regulation of a wide range of complementary therapies, this work primarily addresses those $\mathrm{T} \& \mathrm{CM}$ systems and practices that clearly originate in precolonial cultures. This approach permits a thematic emphasis on the historical, political and epistemic factors that continue to impact upon the statutory regulation of such health care approaches. The authors invite discussion from those engaged in various facets of $\mathrm{T} \& \mathrm{CM}$ regulation worldwide, in pursuit of a robust conversation as to the potential advantages and challenges associated with different policy making approaches.

\section{The Current State of T\&CM Professional Regulation}

As of 2012, 56 United Nations member states surveyed had implemented some form of T\&CM professional regulation, whereas 56 others had no such regulations (and 17 did not answer). ${ }^{1}$ The WHO has characterized state healthcare systems worldwide as falling under one of four types with respect to T\&CM: "integrative" systems in which biomedicine and T\&CM are both funded and recognized; "inclusive" systems, where T\&CM has some recognition but is only partially regulated or integrated into public care; "tolerant" systems, where some T\&CM practices are "tolerated by law" but poorly integrated; and biomedically "exclusive" systems, where T\&CM practices are outlawed. ${ }^{3}$

As Dixon has noted, a range of $\mathrm{T} \& \mathrm{CM}$ professional regulatory models have been implemented worldwide. ${ }^{4}$ These include models of "direct government-administered regulation, ... government-sanctioned self-regulation, ... and independent self-regulation." Particular regulations may apply to T\&CM practices (e.g., acupuncture, herbal medicine) or professions as a whole (e.g., Chinese medicine). Regulations may furthermore protect occupational titles (e.g., "Acupuncturist"), and/or restrict particular practices (e.g., acupuncture), for specified groups' usage. T\&CM professional regulations worldwide are most often implemented at the national/federal level, but in some countries are governed at the regional/provincial/state level. ${ }^{4,5}$

\section{The Need for T\&CM Professional Regulatory Guidance}

WHO data from 2012 strongly suggest that state regulators across the globe are seeking additional guidance to assist with the implementation of T\&CM professional regu- lations. ${ }^{1}$ Notably, $88 \%$ and $52 \%$ of nation states surveyed identified a "lack of research data" and a "lack of expertise within national health authorities and control agencies," respectively, as a significant challenge in this regard. Similarly, the majority of nation states surveyed characterized "information sharing on (T\&CM professional) regulatory issues" (81\%) and "national capacity building seminar/workshop on (T\&CM professional) regulations" (75\%) as a priority.

To date, however, "there has been little research into the relative merits of different approaches to the regulation of practitioners of traditional/complementary medicine, particularly against specified objectives." lished training benchmarks for several T\&CM practices and systems (including acupuncture, traditional Chinese, Ayurvedic, and Unani medicine; traditional Thai massage, chiropractic, osteopathy, and naturopathy) to assist regulators in shaping local entry requirements. However, these benchmarks do not address the specific policy making challenges that state actors and practitioner groups may encounter when negotiating professional regulations for T\&CM practitioners. Such challenges, as the authors will discuss, pertain to:

- historical circumstances (and resulting evidentiary tensions) that surround traditional medicine's political subjugation to Western biomedical knowledge systems;

- concurrent clinical and cultural characteristics of traditional medicine systems and practices;

- challenges (and/or incongruity) of accommodating internally diverse indigenous knowledges and practices into models of regulatory standardization;

- intellectual property considerations; and

- the increasing globalization and biomedicalization of traditional medicine.

To optimize the accessible delivery of safe and effective T\&CM care for patients, while attending to the preservation of valuable indigenous knowledges, regulators would optimally address each of these factors, as the authors now discuss.

\section{Impacts of European Colonization}

An important issue that frequently goes unaddressed in scholarly discussions of T\&CM pertains to the historical and ongoing impacts of European colonization on traditional medicine systems and practices across the globe. As documented and discussed elsewhere, ${ }^{6-9}$ traditional medicine treatments and practices have long been subjugated, devalued, co-opted, and in some cases decimated across the globe within the context of European colonization. Still today, many indigenous healthcare systems remain under threat due to colonization's impacts. ${ }^{10}$

Biomedicine's globalized dominance, as Hollenberg and Muzzin have elaborated, is far less the result of biomedical science's evidenced efficacy than it is a feature of the ongoing sociopolitical subordination of precolonial indigenous knowledge systems and related healthcare practices. ${ }^{11}$ Traditional medicine continues to be in widespread use, and in many jurisdictions (particularly in the global South) represents the "mainstay of healthcare delivery." " However, considerable political, research, economic, and institutional capital continues to sustain biomedicine's pre-eminence in state healthcare systems worldwide. ${ }^{11,12}$ Regardless, indigenous systems of medical knowledge remain important 
resources not only within their specific cultural contexts but also as "critical alternative models for resolving health crises on a global scale where biomedical and technological solutions fall increasingly short.",2

The study and reframing of traditional medicine approaches using biomedical conceptual frameworks and language have been used, for many decades, arguably as a strategy to increase their perceived legitimacy within biomedically dominant healthcare systems. ${ }^{12}$ This has included the increased adoption of biomedical subject areas in the curricula of institutionalized training programs for codified traditional medicine systems such as Chinese medicine, Ayurveda, and Unani, as well as an increasing body of biomedical-style research conducted on particular traditional medicine therapies.

\section{Healthcare's Culturally Situated Hybrid Character}

In line with its globally dominant position, biomedicine is widely and falsely universalized as "culturally neutral."11 Far from being an "unbiased" system of healthcare, biomedicine is itself a cultural artifact, rooted in the European scientific revolution and the linear reductionism of Rene Descartes and his contemporaries. ${ }^{6}$ At the heart of biomedical epistemology are demands for:

uniform and standardized practices, searches for universal causal laws, claims to enact 'value-free' and empirical observation and analysis, and ...assumptions of objectivity, rationality, replicability, comparability, and generalizability. ${ }^{10}$

That said, biomedicine is deeply indebted to indigenous medical systems for many of its therapeutic approaches. As Qi points out, "nearly a quarter of all modern medicines are derived from natural products, many of which were first used in a traditional medicine context." ${ }^{13}$ However, most traditional herbal medicines have undergone considerable recomposition en route to contemporary pharmaceutical usage, in a process that privileges biomedical epistemology while erasing/negating the remedies' indigenous cultural origins and epistemic underpinnings. ${ }^{14}$

In contrast, many traditional medicine systems and practices-distinguished by precolonial epistemic frameworks - today remain explicitly entrenched in their indigenous cultural contexts. ${ }^{6,12,15}$ In 2012, for instance, the United Nations Educational, Scientific and Cultural Organization (UNESCO) inscribed "acupuncture and moxibustion of traditional Chinese medicine" on its Representative List of the Intangible Cultural Heritage of Humanity, attesting to its ongoing cultural-as well as clinical-importance. ${ }^{16}$ Nevertheless, traditional medicine systems and practices are not static timeless entities that remain unchanged over centuries or millennia. Rather, they are internally diverse, increasingly globalized healthcare approaches that continue to evolve and hybridize, including in contact with biomedicine, and are thus not only "traditional" but also contemporary. ${ }^{17}$

In developing professional regulatory structures for traditional medicine, policy makers must invariably contend with the distinct epistemologies that underpin these systems and practices, as well as their culturally situated character. As their own study of Chinese medicine and acupuncture's regulation in the province of Ontario, Canada has shown, such regulatory negotiations can present considerable diffi- culty. In defining acupuncture for regulatory purposes, the Ontario government was challenged to determine the degree to which perspectives from traditional Chinese medicine versus biomedicine should be privileged. ${ }^{2}$ Ontario's Chinese medicine regulator furthermore struggled to establish regulatory mechanisms that would not exclude senior East Asian immigrant practitioners from professional entry due to low proficiency in English, Ontario's dominant language. ${ }^{18}$

Although the Ontario case highlights particular challenges associated with regulating traditional medicine practitioners in diasporic context (i.e., outside of the systems' geographies of origin), such challenges are also evident in indigenous cultural settings. For instance, a linguistic policy challenge that notably paralleled Ontario's situation has been reported in China among Uyghur indigenous medicine practitioners, who have long practiced in the Taklamakan desert region of northeastern China. ${ }^{19}$ Uyghur doctors are regulated by the Chinese government and receive state reimbursement for their private and hospitalbased services. ${ }^{1}$ Since 2000 , Uyghur practitioners are required to complete professional licensure examinations in Mandarin, China's politically dominant language. ${ }^{20}$ As in Ontario, these practitioners have raised alarm that their regulator's language policy has compromised the professional entry of a number of senior practitioners, "threatening the future of the tradition."

Such policy making difficulties, the authors argue, point to the need for innovative regulatory structures that address traditional medicine's unique dimensions. This in itself represents a daunting prospect, at times raising questions about the compatibility of contemporary professional regulatory systems with traditional medicine itself, as the authors now discuss.

\section{The Cultural and Epistemic Foundations of Professional Regulation}

As Adams has reviewed, "professional regulation is... a modern phenomenon dating from the nineteenth century" in Europe. $^{21}$ The emergence of contemporary professions has been "linked to the rise of the modern state," the "spread of an industrial-capitalist economy," the increased sociocultural importance of Western science, "urbanization," and "the expansion of the university system," among other factors. As implemented across liberal democracies today, professional regulation-like biomedicine-bears the epistemic hallmarks of contemporary Western thought.

Indeed, uniformity (of training) and standardization (of credentials) are requisite features of contemporary professional regulation. ${ }^{22,23}$ Formal credentials have become ubiquitous requirements for professional entry, even across occupations in which more informal methods of demonstrating competency were previously accepted as evidence of high skill and knowledge. "24 "Evidence-based" biomedical ideals have moreover become increasingly important in health professional regulation, alongside the previous primary emphasis on preventing incompetent or dangerous practice. ${ }^{25}$

Across the globe, however, traditional medicine knowledge continues to be transmitted informally, through family lineages and apprenticeships between senior and junior practitioners, as well as (increasingly) in more formal institutional settings. ${ }^{1}$ Kovach, a Canadian Indigenous scholar, 
has noted that "Indigenous knowledges can never be standardized," 26 due to their inherent internal diversity and living dynamic character. ${ }^{27}$ Indeed, even for an apparently singular traditional medicine practice - such as acupuncture-a wide range of theoretical and practical approaches coexist across Eastern Asia, which carry specific cultural significance for particular communities. ${ }^{28,29}$

Regulators may be accustomed to contending with competing knowledge claims as particular occupational factions lobby for exclusive regulatory privileges. ${ }^{30}$ However, the internal diversity of traditional medicine approaches raises particular regulatory complexities when standardization becomes associated with regulation. Differences among practitioners as to appropriate professional entry requirements may initially appear to regulators or researchers as "acrimony",31 or "organizational weakness and internal fragmentation and competition.", 32 Whether such disagreements may be better attributed to the incongruity of harmonizing genuine differences in practice warrants careful consideration; such a possibility will require unique regulatory mechanisms to effectively address. Yet more serious regulatory considerations may arise if standardized institutional trainings become the primary route to regulate professional entry for traditional medicine providers, as now elaborated.

\section{Standardization and Institutionalization of Traditional Medicine Practice}

Although apprenticeship and family lineage remain important mechanisms of traditional knowledge transmission across the globe, traditional medicine practitioner trainings in many countries have increasingly moved into institutional settings worldwide. ${ }^{1}$ As Langford, ${ }^{33}$ Hardiman, ${ }^{15}$ and Tay$\operatorname{lor}^{34}$ discuss, the standardization and institutionalization of traditional Chinese, Ayurvedic, and Unani systems of medicine have occurred over the last century as deliberate nationalistic responses to colonial conditions and biomedical dominance, meant to increase political capital surrounding indigenous healthcare approaches.

It should not go unnoticed that it is for these very traditional medicine "systems" (an idea that essentializes their variations) that the WHO has produced training benchmarks. These benchmarks provide regulators with detailed parameters around which to implement professional regulations aimed at increasing safety, access, and quality of care and, thus, remain an important policy making resource. It is also important to recognize that the statutory regulation of traditional Chinese medicine, Ayurveda, and Unani medicine in their geographies of origin, paired with state funding for service, provides large numbers of people with safe, accessible, and quality care that is also culturally relevant.

That having been said, the standardization of particular indigenous healthcare approaches may simultaneously serve to exclude, subordinate, and in some cases threaten the longevity of the many diverse bodies of healing knowledge and styles of medical practices endemic to South and East Asian regions and cultures. Lambert has discussed this phenomenon with reference to the statutory regulation of AYUSH (Ayurveda, Yoga and Naturopathy, Unani, Siddha and Homeopathy) in India. There, she documents "selective processes of legitimation... whereby particular traditions of indigenous medicine undergo reformulation into professionalized and accredited knowledge systems." 24 As a result, a wide range of indigenous medical practitioner groups representing noncodified lineages continue to "practice without official sanction," occupying subordinate sociocultural positions amid "hierarchies of legitimacy" resulting from the statutory regulation of other T\&CM groups.

It thus becomes evident that while significant advantages to patients may come from the professional regulation of traditional medicine in some settings, detrimental consequences are likely to result as well. Regulators will need to be alert to the range of unintended consequences that may arise in regulating traditional medicine practitioners-in particular as they may threaten the integrity or longevity of indigenous healthcare lineages.

\section{Professional Regulation as an Intellectual Property Claim}

As the WHO affirms in its recent Traditional Medicine Strategy, "issues related to intellectual property (IP) can have an impact on products, practices, and even practitioners," thus characterizing the protection of traditional medical knowledge as a global regulatory priority. ${ }^{1}$ To date, the issues surrounding intellectual property and the misappropriation of traditional knowledge have been extensively explored in relation to protecting products (such as traditional herbal formulations) from unwarranted patents. $7,35,36$ However, with a few exceptions, ${ }^{2,35}$ little has been said in the literature to date about the regulatory protection of traditional knowledge as it pertains to indigenous healthcare practices.

The authors argue that health professional regulation itself represents a claim to intellectual property, initially by state actors, and ultimately by regulated providers. Depending on the policy mechanisms implemented, professional regulation of traditional medicine practitioners and practices may entrench in law various intellectual property claims over bodies of indigenous knowledge, occupational titles, diagnostic approaches, and treatment practices. If the preservation of traditional knowledge is to be taken as a regulatory goal, as the WHO advocates, policy makers must be alert to the forms that cultural misappropriationin other words, the abuse of indigenous medical intellectual property-may take in professional regulatory context.

Misappropriation is a concept that refers to the unsanctioned harmful extraction and decontextualization of particular cultural elements or practices, from their whole cultural contexts, usually by cultural outsiders. ${ }^{37}$ With reference to traditional medicine, Hollenberg and Muzzin characterize two key forms of misappropriation using the terms "paradigm appropriation" and "paradigm assimilation." In "paradigm appropriation," they explain that:

biomedicine appropriates certain aspects from other healing systems or traditions without fully acknowledging the paradigmatic worldview from which the particular treatment aspect was taken. ${ }^{11}$

"Paradigm assimilation" goes one step further and, as a "predatory" strategy, "reinterprets" a particular healthcare approach from an indigenous system, reframing the approach in biomedical terms. The principles of paradigm appropriation and assimilation are evident in T\&CM regulatory trends across several jurisdictions, both in the indigenous 
geographies of traditional medicine systems and practices and in diaspora.

In several nations of the global South, there has been a trend in recent decades for governments to regulate traditional medicine practitioners as one response to insufficient funding for and availability of primary healthcare. With reference to the WHO's global "Health for All by the Year 2000" strategy, governments would commonly train traditional medicine practitioners to provide basic biomedical services, which these licensed practitioners would then, presumably, deliver alongside traditional medicine care. ${ }^{38,39}$ However, as Torri has observed (with reference to traditional midwives in Mexico), such efforts may be characterized by a reification of biomedical knowledge, subordinating (or even condemning) traditional medicine approaches as inferior or unsafe. ${ }^{38}$ Biomedical providers, in turn, may make few efforts to inform themselves about the healthcare approaches applied by their indigenous medicine counterparts. Such scenarios, taken as a whole, reinforce an ethos of paradigm assimilation that reframes traditional medicine practitioners' value almost entirely in terms of their ability to deliver biomedicalized care. In this process, the uneven power relations between biomedical and indigenous healthcare providers become reinforced.

In the global North, misappropriation has also been evident in the statutory regulation of traditional medicine practitioners and practices, with acupuncture providing a salient example.

In several North American jurisdictions and in Australia, biomedically trained health practitioners (such as physicians, physical therapists, and chiropractors) are permitted to use acupuncture needles within their statutory scope without statutory reference to East Asian medical theory. ${ }^{40,41}$ As Hollenberg and Muzzin note, such practices demonstrate paradigm appropriation in action. ${ }^{11}$ In some cases, paradigm assimilation is further evident when professional certification bodies explicitly disavow the relevance of traditional East Asian medical principles to their members' biomedicalized use of acupuncture-like needling. ${ }^{42}$ The biomedicalization of traditional medicine practices (like acupuncture), and their use by a range of healthcare providers, need not always represent harmful misappropriation, but the potential for such to be occurring should certainly be an issue considered by regulators.

As elsewhere discussed, considerable research remains to be done to better understand the dynamics of misappropriation in the regulation of traditional medicine practitioners and practices. $^{2}$ What is important in this regard is that regulators be alert to the ways in which biomedicine's existing dominance may be exerted, whether intentionally or unintentionally, in professional regulatory processes.

\section{Occupational Self-Interest and the Public Interest}

In some jurisdictions, particular groups of T\&CM practitioners may be eager to "earn" professional regulatory status from the state, hoping to increase their sociopolitical power and economic status. ${ }^{5,31,43-46}$ As scholars of professionalization have long observed, occupational self-interest and competing claims to authority over knowledge are common issues with which regulators are faced across a range of professions. ${ }^{23,30}$ The state, it has been widely ar- gued, should carefully subordinate such self-interest and competing knowledge claims to the interests of the public (represented in the healthcare field as patients). ${ }^{47}$ However, as their own work in the field of Chinese medicine regulation has documented, policy makers accustomed to making decisions from within conceptual and regulatory frameworks that (implicitly or explicitly) privilege biomedical knowledge, may have difficulty "differentiating between, and avoiding conflation of, [T\&CM] stakeholders' epistemic claims and occupational self-interest.",

For practitioner groups to advocate for the centralization of indigenous cultural and epistemic perspectives in T\&CM regulatory parameters should not, the authors argue, be automatically conceived of as professional self-interest. Rather, such advocacy may represent an important effort to protect and preserve traditional knowledge, itself a public interest priority in T\&CM professional regulation. Consideration of such perspectives does not, the authors argue, detract from policy makers' ability to centralize the interests of patients in crafting appropriate T\&CM professional regulations. Rather, as discussed below, such an approach may help to sharpen regulators' focus on creating policies that serve those patients most in need of T\&CM care.

\section{Prioritizing Key Publics in T\&CM Professional Regulation}

Patients across the globe avail themselves of T\&CM care for diverse reasons. In many places, in particular in the global South, traditional medicine care represents the most geographically and/or financially accessible form of healthcare (even when the patient might prefer biomedical services). ${ }^{48}$ Moreover, both in the South and the North, indigenous medicine practitioners provide sought-after culturally situated care whose underlying worldview and epistemic concepts align with those of its primary users. ${ }^{1}$ In global North settings, T\&CM care has also come to represent a supplement (or in some cases a replacement for) statefunded biomedical care for those affluent enough to pay for it. ${ }^{48}$ Among such users, many are disillusioned with biomedicine's mechanistic, symptom-driven approach and are attracted to the "holistic," so-called "natural," and patientcentered experience represented by many forms of T\&CM. ${ }^{49}$ Worldwide, women are the predominant users of $\mathrm{T} \& \mathrm{CM}$ in all its forms ${ }^{48}$ (a point that warrants further unpacking in regulatory context).

In considering whether or how to regulate $\mathrm{T} \& \mathrm{CM}$ in a particular jurisdiction, it is essential that patients most in need of safe, effective primary healthcare be prioritized to ensure that they receive regulation's benefits. In the global South, such patients are likely to be poor and without ready access to other health services. In the North, regulators should give particular consideration to patients known to be underserved by mainstream biomedicine, as well as members of ethnic communities for whom particular forms of T\&CM may represent an important source of culturally appropriate care.

The implementation of professional regulations in any setting will inevitably exclude some providers, thus impacting their existing patients. In some cases, this may prove advantageous by deterring unskilled or unscrupulous practitioners from practice. Regardless, the degree to which a particular professional regulatory approach fosters increased 
accessibility of T\&CM care for a jurisdiction's most socially disadvantaged patients should be a primary measure of the policy's effectiveness.

\section{The Need for Professional Regulatory Guidelines for Traditional Medicine}

As discussed in this work, the statutory regulation of traditional medicine practitioners and practices presents a set of distinct policy challenges that arise in large part from the worldwide legacy of European colonization. Biomedicine's disproportionate power in global health systems and the predominance of biomedical epistemology and discourse in health professional regulatory structures make regulation of traditional medicine providers a complex prospect rife with potential pitfalls. To uphold the promise of increased safety, access to, and quality of traditional medicine care advocated by the WHO, regulators must attend vigilantly to the policy challenges at hand, whether epistemic or structural. It is evident, however, that few resources currently exist to help regulators in this regard. Nor does there yet exist a substantial body of scholarship to rigorously examine the dynamics of such challenges.

As the authors have elsewhere argued, the principle of regulatory equity, that is, an emphasis on fair outcomes that seek to redress historically situated inequalities, should be centralized by policy makers as they craft innovative frameworks to address their particular health system needs. ${ }^{2}$ Traditional knowledge protection, and the prevention of further misappropriation of indigenous medical practice, should also be prioritized, giving importance to traditional medicine's culturally situated and internally diverse character. Additional work will be needed to elaborate upon how these principles may be operationalized, with reference to other core public interest considerations such as safety, accessibility, and healthcare quality. In the interim, the authors welcome feedback from regulators, practitioners, researchers, and others, to foster increased dialogue and scholarship in the emerging field of traditional medicine professional regulatory studies.

\section{Author Disclosure Statement}

No competing financial interests exist.

\section{References}

1. World Health Organization. WHO Traditional Medicine Strategy 2014-2023. Geneva: World Health Organization, 2013. Online document at: www.who.int/medicines/publications/ traditional/trm_strategy14_23/en/. Accessed September 10, 2014.

2. Ijaz N, Boon H, Muzzin L, Welsh S. State risk discourse and the regulatory preservation of traditional medicine knowledge: The case of acupuncture in Ontario, Canada. Soc Sci Med 2016;170:97-105.

3. World Health Organization. WHO Traditional Medicine Strategy 2002-2005. Geneva: World Health Organization. Online document at: www.wpro.who.int/health_technology/ book_who_traditional_medicine_strategy_2002_2005.pdf. Accessed March 10, 2014.

4. Dixon A. Regulating complementary medical practitioners: An international review. King's Fund, 2008. Online document at: www.kingsfund.org.uk/sites/default/files/Research\%20 summary1.pdf. Accessed April 24, 2016.

5. Ijaz N, Boon H, Welsh S, Meads A. Supportive but 'worried': Perceptions of naturopaths, homeopaths and Chinese medicine practitioners through a regulatory transition in Ontario, Canada. BMC Complement Altern Med 2015;15:312-325.

6. Harding S. Is Science Multicultural? Postcolonialisms, Feminisms and Epistemologies. Bloomington, IN: Indiana University Press, 1998.

7. Shiva V. Biopiracy: The Plunder of Nature and Knowledge. Toronto, Canada: Between the lines, 1997.

8. Battiste M. Post-colonial remedies for protecting Indigenous knowledge and heritage. In: Tripp P, Muzzin L, eds. Teaching as Activism: Equity Meets Environmentalism. Kingston, Canada: McGill-Queen's University Press, 2005.

9. Banerji D. The place of indigenous and Western systems of medicine in the health services of India. Soc Sci Med 1981; 15A:109-114.

10. Oliver $\mathrm{S}$. The role of traditional medicine practice in primary health care within Aboriginal Australia: A review of the literature. J Ethnobiol Ethnomed 2013;9:1-8.

11. Hollenberg D, Muzzin L. Epistemological challenges to integrative medicine: An anti-colonial perspective on the combination of complementary/alternative medicine with biomedicine. Health Sociol Rev 2010;19:34-56.

12. Janes $C$. The health transition, global modernity and the crisis of traditional medicine: The Tibetan case. Soc Sci Med 1999;48:1803-1820.

13. Qi Z. Global situation and implementation of WHO TM strategy 2014-2023. World Health Organization, 2017. Online document at: www.paho.org/hq/index.php?option= com_docman\&task=doc_view \&Itemid=270\&gid=40651\& lang=en. Accessed October 10, 2017.

14. Ijaz N. A post-colonial feminist analysis of two popular complementary medicine hot flash remedies. In: Torri $\mathrm{C}$, Hornosty J (eds). Complementary, Alternative \& Traditional Medicine: Prospects and Challenges for Women's Reproductive Health. Toronto: Women's Press, 2017.

15. Hardiman D. Indian medical indigeneity: From nationalist assertion to the global market. Soc Hist 2009;34:263-283.

16. United Nations Scientific and Cultural Organization (UNESCO). Acupuncture and moxibustion of traditional Chinese medicine. UNESCO, 2010. Online document at: www.unesco.org/culture/ich/index.php?lg=en\&pg=00011 $\&$ RL=00425. Accessed February 10, 2014.

17. Marsland R. The modern traditional healer: Locating 'hybridity' in modern traditional medicine, southern Tanzania. J South Afr Stud 2008;33:751-765.

18. Ijaz N. Regulating Traditional Medicine Professionals in the Public Interest: A Case Study of Chinese Medicine and Acupuncture Regulation in Ontario, Canada. Toronto: University of Toronto, TSpace, 2017.

19. Abdukadir A, Dubrovin D, Amat N, et al. The origins of Uyghur medicine: Debates and perspectives. JTCMS 2015; 2:217-226.

20. Turdush R, Juma M, Vandenbrink R. Uyghur traditional medicine doctors tested in Chinese. Radio Free Asia, 2013. Online document at: www.rfa.org/english/news/uyghur/ medicine-09242013202312.html. Accessed August 22, 2014.

21. Adams T. The changing nature of professional regulation in Canada, 1867-1961. Soc Sci Hist 2009;33:217-243.

22. Maroto ML. Professionalizing body art: A marginalized occupational group's use of informal and formal strategies of control. Work Occup 2011;38:101-138. 
23. Abbott A. The System of Professions. Chicago, IL: University of Chicago Press, 1988.

24. Lambert H. Medical pluralism and medical marginality: Bone doctors and the selective legitimation of therapeutic expertise in India. Soc Sci Med 2012;74:1029-1036.

25. Madison K. Donabedian's legacy: The future of health care quality law and policy. Indiana Health Law Rev 2012;10: 325-364.

26. Kovach M. Indigenous Methodologies, Characteristics, Conversations and Contexts. Toronto, Canada: University of Toronto Press, 2009.

27. Hsu E. Innovation in Chinese Medicine. Cambridge, United Kingdom: Cambridge University Press, 2001.

28. Hsu E. The Transmission of Chinese Medicine. Cambridge, United Kingdom: Cambridge University Press, 1999.

29. Scheid V. Chinese Medicine in Contemporary China. Durham, NC: Duke University Press, 2002.

30. Witz A. Professions and Patriarchy. London, United Kingdom: Routledge, 1992.

31. Welsh S, Kelner M, Wellman B, Boon H. Moving forward? Complementary and alternative practitioners seeking selfregulation. Sociol Health Illn 2004;26:216-241.

32. Freidin B. Acupuncture worlds in Argentina: Contested knowledge, legitimation processes, and everyday practices. ProQuest, UMI Dissertations Publishing, Brandeis University, 2007:3290969.

33. Langford JM. Fluent Bodies: Ayurvedic Remedies for Postcolonial Imbalances. Durham, NC: Duke University Press, 2002.

34. Taylor K. Chinese Medicine in Early Communist China, 1945-1963: A Medicine of Revolution. New York, NY: Routledge Curzon, 2005.

35. Srinivas KR. Intellectual property rights and traditional knowledge: The case of yoga. Econ Political Wkly 2007; 42:2866-2871.

36. World Health Organization, World Intellectual Property Organization, World Trade Organization. Promoting access to medical technologies and innovation: Intersections between public health, intellectual property and trade. Geneva, 2013. Online document at: www.wto.org/english/ res_e/booksp_e/pamtiwhowipowtoweb13_e.pdf. Accessed June 24, 2015.

37. Brown M. Heritage trouble: Recent work on the protection of intangible cultural property. IJCP 2005;12:40-61.

38. Torri C. Traditional midwives in Tamaulipas: The difficult negotiation between traditional child-birth knowledge and the biomedical system in Mexico. Womens Health Urban Life 2012;11:42-63.
39. Kelly T. Contemplating collaboration: Traditional medicine, biomedicine, and co-ordination of health care in Cameroon. Africa Research Institute, 2015. Online document at: www .africaresearchinstitute.org/newsite/blog/contemplating-collab oration-traditional-medicine-biomedicine-and-co-ordinationof-health-care-in-cameroon/. Accessed October 29, 2017.

40. Dommerholt J. Dry needling-Peripheral and central considerations. J Man Manip Ther 2011;19:223-237.

41. Janz S, Adams J. Acupuncture by another name: Dry needling in Australia. AJACM 2011;6:3-11.

42. American Physical Therapy Association. Physical therapists and the performance of dry needling: An educational resource paper. APTA Department of Practice and APTA State Government Affairs, 2012. Online document at: www.apta .org/StateIssues/DryNeedling/ResourcePaper/. Accessed July $16,2016$.

43. Gilmour J, Kelner M, Wellman B. Opening the door to complementary and alternative medicine: Self-regulation in Ontario. Law Policy 2002;24:149-174.

44. Cant S, Sharma U. The reluctant profession-Homoeopathy and the search for legitimacy. Work Employ Soc 1995;9: 743-762.

45. Canaway R. A culture of dissent: Australian naturopaths' perspectives on practitioner regulation. Complement Health Pract Rev 2009;13:136-152.

46. Wiese M, Oster C. 'Becoming accepted': The complementary and alternative medicine practitioners' response to the uptake and practice of traditional medical therapies by the mainstream health sector. Health 2010;14:415-433.

47. Saks M. Professions and the Public Interest: Medical Power, Altruism and Alternative Medicine. London: Routledge, 1995.

48. Keshet Y, Simchai D. The 'gender puzzle' of alternative medicine and holistic spirituality: A literature review. Soc Sci Med 2014;113:77-86.

49. Kaptchuk T, Eisenberg D. The persuasive appeal of alternative medicine. Ann Intern Med 1998;129:1061-1065.

Address correspondence to: Nadine Ijaz, PhD

Leslie Dan Faculty of Pharmacy University of Toronto 144 College Street Toronto, ON M5S $3 M 2$

Canada

E-mail: nadineijaz@gmail.com 